\title{
Impacto da exposição gengival na estética do sorriso
}

\author{
Impact of gingival exposure on smile aesthetics
}

\author{
Nicole Fornari Sígolo ${ }^{1^{*}} \bullet$, Emanuel Silva $\operatorname{Rovai}^{2} \bullet$ Valdineia Maria Tognetti $^{3} \bullet$ \\ ${ }^{1}$ Universidade São Francisco, Bragança Paulista, São Paulo, Brasil. ${ }^{2}$ Universidade Estadual Paulista, São José dos Campos, São Paulo, \\ Brasil. ${ }^{3}$ Universidade São Francisco, Bragança Paulista, São Paulo, Brasil. *Autor para correspondência. E-mail: \\ nicolesigolo@gmail.com
}

\begin{abstract}
Resumo: Introdução: Atualmente a busca pela estética é crescente nos consultórios odontológicos. A queixa da condição gengival do sorriso é um fator que causa grande insatisfação na estética do sorriso e tem impacto negativo na percepção dos outros, afetando a vida social, pessoal e profissional dos pacientes. Para um resultado satisfatório no tratamento dessa condição é necessário traçar um plano de tratamento individualizado para cada paciente, fazendo um diagnóstico correto da etiologia do sorriso gengival, bem como o tratamento mais adequado para cada caso. O objetivo do presente estudo foi realizar uma revisão de literatura nos aspectos que influenciam a estética do sorriso, as diversas etiologias do sorriso gengival e as possibilidades de tratamento para cada caso. Revisão: As bases de dados utilizadas para a pesquisa bibliográfica foram: PubMed, Google Acadêmico e Scielo com publicações científicas datadas entre os anos de 1965 a 2020. Discussão: Os estudos mostraram que a queixa da condição de sorriso gengival é um fator que causa grande insatisfação na estética do sorriso e gera um impacto negativo na percepção de terceiros, afetando a vida social, pessoal e profissional dos pacientes. Além disso, o sorriso gengival apresenta uma etiologia diversa, que pode se apresentar isolada ou combinada, dessa forma, o tratamento dessa condição exige um trabalho multidisciplinar. Considerações finais: Para alcançarmos um resultado satisfatório no tratamento dessa condição, é necessário traçar um plano de tratamento individualizado para cada paciente, realizando um correto diagnóstico da etiologia do sorriso gengival e posteriormente, optar pelo tratamento mais adequado para cada caso.
\end{abstract}

Palavras-chave: crescimento excessivo da gengiva, estética dentária, periodontia, sorriso.

\begin{abstract}
Introduction: Currently, the search for aesthetics is growing in dental offices. The complaint of the gingival condition of the smile is a factor that causes great dissatisfaction in the aesthetics of the smile and has a negative impact on the perception of others, affecting the social, personal and professional life of the patients. For a satisfactory result in the treatment of this condition, it is necessary to outline an individualized treatment plan for each patient, making a correct diagnosis of the etiology of the gingival smile, as well as the most appropriate treatment for each case. The aim of the present study was to carry out a literature review on the aspects that influence the aesthetics of the smile, the different etiologies of the gingival smile and the treatment possibilities for each case. Review: The databases used for the bibliographic search were: PubMed, Google Scholar and Scielo with scientific publications dated between 1965 and 2020. Discussion: Studies have shown that the complaint of gingival smile is a factor that causes a great deal dissatisfaction in the aesthetic of the smile and generates a negative impact on the perception of others, affecting the social, personal and professional lives of patients. In addition, the gingival smile has a different etiology, which can be isolated or combined, so the treatment of this condition requires multidisciplinary work. Final considerations: In order to achieve a satisfactory result in the treatment of this condition, it is necessary to draw up an individualized treatment plan for each patient, making a correct diagnosis of the etiology of the gingival smile and later, choosing the most appropriate treatment for each case.
\end{abstract}

Keywords: periodontics, gingival overgrowth, smiling esthetics, dental.

\section{Introdução}

A estética vem ganhando mais espaço a cada dia nos consultórios odontológicos e para alcançarmos resultados satisfatórios é necessário haver harmonia e simetria entre as estruturas anatômicas que compõem o sorriso (Rocha Neto \& Damin, 2009). O ato de sorrir é uma das mais importantes expressões faciais e fundamental para demonstrar emoções (Peck et al., 1992). Neste contexto, um aspecto que vem gerando muito interesse em relação à estética é a quantidade de exposição gengival no sorriso (Sarver \& Ackerman, 2003). 
Para atingir bons resultados no tratamento do sorriso gengival, precisamos analisar diversos fatores e realizar um plano de tratamento individual para cada paciente, já que quando tratamos de estética há uma variação no conceito de beleza para cada indivíduo e por isso a opinião do paciente deve ser respeitada. Outra variação no conceito de beleza encontra-se entre pacientes e dentistas, os profissionais no ramo da estética criam um olhar extremamente crítico e rigoroso frente aos padrões e que muitas vezes não se encaixam nas expectativas do paciente (Pires et al., 2010), entretanto, mesmo com essa diferença na percepção, estudos mostram que para ambos (profissionais e leigos) o sorriso influencia na percepção da beleza. Além disso, esses conceitos devem sempre estar em equilíbrio com o princípio de saúde.

Em relação a quantidade de gengiva exposta, a harmonia, a simetria do contorno, a arquitetura e a cor dos tecidos são fatores que se demonstram em evidência na Periodontia atualmente (Morais et al., 2010). Sorrir, é uma forma de transmitir boas emoções e influencia positivamente na percepção de terceiros quando esta expressão é manifestada (Kraut \& Johnston, 1979), entretanto, estudos mostram que o sorriso gengival pode provocar impressões negativas em relação a atratividade, amabilidade, confiabilidade, inteligência e autoconfiança (Malkinson et al., 2013). Sendo assim, podemos perceber o quanto o sorriso é importante na vida de cada indivíduo e entender que ao realizarmos qualquer tratamento que gere mudança nesse aspecto estamos ocasionando também uma mudança social (Neto et al., 2017).

As causas do sorriso gengival são variáveis, sendo muitas vezes resultado de diversos fatores e o tratamento pode envolver, dependendo do diagnóstico, terapia ortodôntica, periodontal e cirúrgica (Geron \& Atalia, 2005). Entre as diversas especialidades da Odontologia, a Periodontia exerce uma função essencial no quesito estética, pois engloba o tratamento de estruturas essenciais na construção da harmonia do sorriso (Garber \& Salama,1996; Saba-Chujfi \& Santos-Pereira, 2007)

Dessa forma, o presente estudo tem como objetivo realizar uma revisão de literatura nos aspectos para avaliação do sorriso gengival na estética do sorriso, suas etiologias e possibilidades de tratamento dessa condição a partir do seguinte questionamento: Qual o impacto do sorriso gengival na estética do sorriso?

\section{Revisão}

O presente estudo foi realizado a partir de uma revisão de literatura, baseado em livros e publicações científicas, entre os anos de 1965 a 2020, relacionando os seguintes descritores de pesquisa: Periodontia, Crescimento excessivo da gengiva, Sorriso e Estética dentária. As bases de dados utilizadas para a pesquisa bibliográfica foram: PubMed, Google Acadêmico e Scielo, nos idiomas português e inglês. Estudo in vitro e em animais foram excluídos do estudo. A análise qualitativa dos estudos foi realizada a partir de estudos que apresentassem o impacto do sorriso gengival na estética do sorriso, tanto para o paciente quanto para terceiros, assim como um tratamento do sorriso gengival baseado em sua etiologia, e a análise quantitativa foi realizada quanto aos resultados obtidos no desfecho primário, como otimização da estética do sorriso, melhora da percepção por terceiros e diagnóstico e tratamento multidisciplinares que contribuíram para a harmonização de todas as estruturas faciais potencializando o resultado estético.

\section{Estética do sorriso}

Estudos constatam que o sorriso modifica a percepção de terceiros e diminuiu o impacto do tipo facial, ou seja, podemos considerar que o sorriso afeta a estética percebida em diferentes padrões faciais (Batwa, 2018). Ao analisar na literatura, encontramos diversos aspectos a serem considerados na estética do sorriso, dentre eles podemos citar princípios estéticos e funcionais, que englobam características relacionadas a face, aos dentes, a gengiva e ao lábio (Chiche \& Pinault, 1994). Na análise facial, deve-se levar em consideração que assimetrias de até 3\% em distâncias lineares não comprometem o resultado final, mas valores acima deste já tornam-se evidentes (Lu, 1965). Diante dessas informações, podemos perceber que a estética do sorriso não está ligada somente a um fator e não pode ser classificada por apenas um parâmetro, a harmonia de todos os fatores é fundamental para um resultado satisfatório e mais importante do que a análise de pontos isolados.

$\mathrm{Na}$ análise facial, é importante relacionar os dentes, a gengiva e os lábios com os aspectos faciais do paciente, harmonizando o rosto como um todo. Para definir parâmetros no diagnóstico, utiliza-se linhas horizontais e verticais como referência: a linha ofríaca (sobrancelhas), a linha interpupilar e a linha da comissura labial, sendo que a linha média deve estar situada perpendicularmente a estas. Caso não haja paralelismo entre as linhas horizontais, deve-se escolher, junto com o paciente, qual dessas linhas irá ser usada como orientação. Posteriormente, o rosto do paciente é dividido em terços, sendo terço superior (linha do cabelo até a linha ofríaca), terço médio (linha ofríaca até a linha alar) e o terço superior da linha alar até a 
ponta do mento. Analisar esses fatores, auxilia no processo de diagnóstico da etiologia do sorriso gengival, como por exemplo, o crescimento maxilar excessivo, que apresenta o terço inferior maior em relação aos outros (Neto et al., 2017).

Além dos conceitos técnicos, não podemos esquecer que a estética é uma questão subjetiva, sendo assim, o que muitos autores dizem ser o "ideal" nem sempre corresponde as necessidades, possibilidades e desejos do paciente. Por essas e outras razões, todos os detalhes do tratamento devem ser muito bem claros e explicados ao paciente, para não gerar expectativas fora da realidade (Rivera et al., 2000). Deve-se refletir que é ilógico criar um padrão estético em uma população mundial com bilhões de pessoas, com as mais diversas características e culturas, é preciso respeitar a singularidade de cada indivíduo em quaisquer aspectos (Owens et al., 2002).

Ao realizar a avaliação clínica nos pacientes, alguns aspectos são fundamentais para serem observados, que serão abordados a seguir:

Distância interlabial em repouso; quando é normal (1-3mm), a causa do sorriso gengival é considerada, principalmente, de origem muscular. Ao apresentar-se com valores aumentados ( $>3 \mathrm{~mm})$, são na maioria das vezes originadas de desarmonias dentofaciais, como o excesso vertical maxilar e/ou protusão dos incisivos superiores, podendo ou não, estar associado a variações anatômicas e/ou funcionais do lábio superior (Peck et al., 1992; Pascotto \& Moreira, 2005).

Exposição dos incisivos superiores durante o repouso e a fala; essa característica apresenta-se com valores distintos entre os gêneros, no sexo feminino varia de $2 \mathrm{~mm}$ a $4,5 \mathrm{~mm}$, enquanto no sexo masculino é de $1 \mathrm{~mm}$ a $3 \mathrm{~mm}$. Quando há uma exposição maior do que o padrão, alguns fatores estão ligados: padrão facial dolicofacial, lábio superior curto, extrusão dos incisivos e/ou excesso vertical maxilar, sendo que nesses dois últimos, o planejamento da correção deve basear-se no grau de exposição dos incisivos que pretende-se manter. O tratamento do sorriso gengival em pacientes que têm possibilidade de intrusão dos incisivos, não apresenta muitas dificuldades no planejamento ortodôntico e da ortocirurgia, porém, quando há boa exposição dos incisivos no repouso e na fala é necessário um plano de tratamento mais delicado (Peck et al., 1992; Sarver \& Ackerman, 2003).

Arco do sorriso; nesse quesito, para ter um sorriso atraente e jovial a curvatura do arco do sorriso deve posicionar-se paralelamente a margem superior do lábio inferior. Nas mulheres, essa curvatura é mais acentuada e nos homens mais plana. O padrão braquifacial possuiu o arco do sorriso mais plano em comparação com o mesofacial e dolicofacial (Peck et al., 1992). Se esse aspecto não for examinado, há possibilidade de ter um resultado mais achatado da curvatura desfavorecendo a estética (Sarver, 2004).

Proporção largura/comprimento dos incisivos superiores; a largura dos incisivos centrais superiores deve ser de $80 \%$ do seu comprimento, sendo aceita de $65 \%$ a $85 \%$, enquanto nos incisivos laterais varia em torno de 70\%, essa proporção se diz respeito ao "padrão ouro" na estética do sorriso (Sarver, 2004; Levin, 1978; Kokich et al., 1984). Nos casos de sorriso gengival, o comprimento dos dentes anteriores superiores deve ser avaliado, se apresentarem coroas muito curtas podemos pensar em duas possíveis causas: Altura reduzida das bordas incisais por fratura/atrição ou aumento de tecido gengival, que podem ter etiologias diversas (Garber \& Salama, 1996; Borghetti, 2002).

Características morfofuncionais do lábio superior; o comprimento médio do lábio superior nas mulheres é em média 20mm, e nos homens 24mm (Burstone, 1967) e mesmo que esse fator pareça estar relacionado com uma exposição maior da gengiva, estudos demonstram que o mesmo pode não ter relação direta com esta ocorrência (Peck et al., 1992). Entretanto, em casos de excesso vertical maxilar, que apresentam tamanho normal ou acentuado do lábio superior, há um desafio para correção do sorriso gengival, pois limita a intrusão dos incisivos (Peck et al., 1992; Cohen, 2008).

Esse "checklist estético", auxilia o profissional em um correto diagnóstico e possibilita a avaliação das características individuais de cada paciente garantindo o registro de informações fundamentais para o plano de tratamento (Seixas, Costa-Pinto e Araújo, 2011).

\section{Sorriso gengival e etiologia}

A condição clínica de sorriso gengival é considerada quando há $2 \mathrm{~mm}$ ou mais de gengiva exposta ao sorrir relacionada a uma "linha de sorriso alta", tendo prevalência em $10 \%$ da população, $14 \%$ no sexo feminino e 7\% no sexo masculino (Tjan \& Miller, 1984). A maioria dos cirurgiões dentistas consideram que ao sorrir, o lábio superior deve posicionar-se ao nível da margem gengival dos incisivos centrais superiores (Legan \& Burstone, 1980; Levine \& Mcguire, 1997; Marckley, 1993), em contrapartida têm-se o conhecimento de que 
uma certa quantidade de gengiva exposta é esteticamente aceitável e até considerada característica que agrega aspecto jovial (Arnett \& Bergman, 1993; Fowler, 1999; Zachrisson, 1998). Uma informação que acrescenta muita relevância nesse contexto é o fato de que a idade e o sexo interferem na altura do sorriso, demonstrando um grau de autocorreção no sorriso gengival ao longo dos anos, principalmente no sexo masculino (Cosendey, 2008; Vig \& Brundo, 1978; Desai et al., 2009). Todas as informações levam-nos a acreditar que a desarmonia causada pelo excesso de tecido gengival está relacionada em maior ou menor grau com a tonicidade muscular (Duarte et al., 2009).

Para elaborarmos um correto plano de tratamento, é preciso conhecer as etiologias dessa condição que podem ser divididas de acordo com os seguintes fatores: Esqueléticos (Crescimento vertical da maxila excessivo; discrepâncias maxilares); Dentais (Coroa clínica curta; Extrusão de incisivos); Labial (Lábio superior curto; Hipermobilidade do lábio superior) ou Periodontais (Erupção passiva ou ativa alterada; Hiperplasia gengival), essas etiologias podem apresentar-se isoladas ou combinadas e a partir do correto diagnóstico, o tratamento pode incluir um ou mais procedimentos (Garber \& Salama, 1996; Silva et al., 2013; Kumar et al., 2007; Zangrando et al., 2017). A ortodontia contemporânea defende a importância de detectar os pontos estéticos favoráveis do sorriso para preservá-los após realizar os tratamentos dentofaciais, ou seja, ao realizar um diagnóstico adequado da etiologia do sorriso gengival podemos traçar a melhor forma de corrigi-lo sem causar danos a outras estruturas que compõe a estética do sorriso (Sarver \& Ackerman, 2003).

\section{Erupção Passiva e Ativa Alterada}

Dentre as mais diversas etiologias do sorriso gengival, temos a Erupção Passiva e Ativa Alteradas. Antes de definir esses dois conceitos, devemos ter em mente que a erupção não termina quando os dentes encontram com seus antagonistas, ela é contínua durante a vida, a depender de diversos fatores relacionados. A Erupção Ativa refere-se a movimentação dentária no sentido da oclusão, para fora do osso alveolar e a Erupção Passiva refere-se ao movimento da gengiva no sentido apical até atingir sua posição correta (próxima a junção cemento-esmalte, JCE), esses processos são fisiológicos e desejados e só apresentam problemas quando acontecem de forma alterada (Neto et al., 2017; Garber \& Salama, 1996; Borghetti, 2002; Newman et al., 2016; Coslet et al., 1977). Para alcançar o correto diagnóstico e posteriormente decidir a correção cirúrgica mais adequada em cada caso, abordaremos a Erupção Passiva Alterada a partir da classificação feita por Coslet et al. (1977), (Tabela 1), essa avaliação é fundamental para a individualização do plano de tratamento, auxiliando na identificação das limitações da técnica e expectativas do paciente (Neto et al., 2017).

Tabela 1. Classificação da Erupção Passiva Alterada. Adaptado de Coslet et al. (1977).

\begin{tabular}{ccc}
\hline $\begin{array}{c}\text { Em relação à altura } \\
\text { gengival }\end{array}$ & $\begin{array}{c}\text { Tipo I: quantidade } \\
\text { excessiva de tecido } \\
\text { queratinizado (da margem } \\
\text { gengival à junção } \\
\text { mucogengival) }\end{array}$ & $\begin{array}{c}\text { Tipo II: faixa de tecido } \\
\text { queratinizado } \\
\text { normal(consideramos de } \\
\text { aproximadamente } 3 \mathrm{~mm} \text { ) }\end{array}$ \\
\hline Em relação à & $\begin{array}{c}\text { Subtipo A: distância da } \\
\text { crista alveolar à JEC de }\end{array}$ & $\begin{array}{c}\text { Subtipo B: crista alveolar ao } \\
\text { nível da JEC. }\end{array}$ \\
alveôncia da crista & aproximadamente 2 mm & \\
\hline
\end{tabular}

Quando a proporção da largura e da altura do dente for maior que 80\% (dente curto), a Erupção Passiva Alterada é a causa do Sorriso Gengival, pois este apresenta maior cobertura de tecido gengival. $\mathrm{O}$ tratamento dessa condição é traçado a partir da sua classificação, sendo: Tipo I- Subtipo A (altura da gengiva queratinizada maior ou igual a $4 \mathrm{~mm}$ com o espaço biológico preservado), onde o tratamento proposto é a gengivectomia; Tipo I- Subtipo B (altura da gengiva queratinizada maior ou igual a $4 \mathrm{~mm}$ e o espaço biológico apresentando um valor de até $3 \mathrm{~mm}$ ), nesse caso o tratamento indicado seria uma combinação de gengivectomia com osteotomia; já no Tipo II- Subtipo A (altura da gengiva queratinizada até $3 \mathrm{~mm}$ com o espaço biológico preservado), a melhor opção é a cirurgia de Retalho Posicionado Apicalmente; por fim, temos a combinação do Tipo II- Subtipo B (altura da gengiva queratinizada até $4 \mathrm{~mm}$ e espaço biológico até $3 \mathrm{~mm}$ ), 
nessa situação partimos para a indicação do tratamento combinando uma Osteotomia com a cirurgia de Retalho Posicionado Apicalmente (Pires et al., 2010; Neto et al., 2017; Garber \& Salama, 1996; West, 2008).

Já a Erupção Ativa Alterada, é regulada pelo fenômeno da atrição, onde a erupção acontece para equilibrar a perda de tecido dental nesse processo com o objetivo de manter a relação adequada entre a coroa clínica e a raiz clínica e a manutenção da dimensão vertical dos dentes, evitando uma desproporção no comprimento que poderia causar sobrecarga aos tecidos periodontais (Newman et al., 2016).

\section{Tratamento}

Como as etiologias do sorriso gengival são múltiplas e podem se manifestar com uma ou mais causas relacionadas, o tratamento dessa condição também pode apresentar uma ou diversas abordagens, envolvendo, muitas vezes, um trabalho interdisciplinar. A partir do diagnóstico, é preciso traçar um plano de tratamento individualizado para cada caso, dentre as técnicas utilizadas para esse fim podemos destacar as cirurgias periodontais, as cirurgias ortognáticas, tratamento ortodôntico, cirurgias mucogengivais e aplicação de medicamentos, que serão discutidas posteriormente (Garber \& Salama, 1996; Silva et al., 2013; Polo, 2008). Uma informação importante no planejamento cirúrgico, é o fato de que ao realizar a queixa da condição de sorriso gengival, o que realmente causa insatisfação na estética para o paciente é a alteração na forma dos dentes e o impacto negativo que essa proporção alterada de gengiva/dente causa, sendo assim, ressalta-se a importância do cuidado no contorno gengival e adequada harmonia em todos esses quesitos (Newman et al., 2016).

Um critério para dar início ao plano de tratamento é a proporção da coroa clínica, quando esta apresentase favorável (entre $75 \%$ e $80 \%$ ), a etiologia do sorriso gengival é a hipermobilidade do lábio e/ou crescimento maxilar excessivo, sendo descartada a necessidade de cirurgias periodontais para redefinir a margem gengival e restaurações com finalidade de correção estética nas coroas. Nesses casos, com valores $\leqslant 4 \mathrm{~mm}$ de exposição gengival a intrusão ortodôntica está indicada, mas para situações onde há valores $>4 \mathrm{~mm}$ com exposição acentuada das incisais em posição de repouso, partimos para a indicação de uma cirurgia ortognática (Le Fort I). Porém, se não houver excesso da exposição das incisais em repouso as possibilidades de tratamento são diferentes, sendo indicada uma aplicação de Toxina Botulínica Tipo A ou uma cirurgia de reposicionamento labial, já que a causa do sorriso gengival nessa situação está ligada somente a hipermobilidade do lábio superior. Por outro lado, quando esses valores se apresentarem menores, ou seja, uma proporção da coroa clínica desfavorável com $<70 \%$ (coroa clínica curta), o tratamento indicado é a intrusão ortodôntica (Neto et al., 2017; Garber \& Salama, 1996; West, 2008).

Em contrapartida, em casos onde a proporção da coroa clínica é desfavorável, as variações no tratamento aumentam. Quando a proporção é maior que $80 \%$ (dente curto), a causa está relacionada com a Erupção Passiva Alterada e o tratamento já foi discutido anteriormente. Mas quando a proporção é menor que $75 \%$, podemos nos deparar com as seguintes condições: Excesso de crescimento maxilar vertical que pode estar associado a uma exposição da gengiva maior que $4 \mathrm{~mm}$ e exposição da incisal excessiva em repouso, onde o tratamento proposto é a de uma Cirurgia Ortognática com osteotomia Le Fort I ou associado a uma exposição da gengiva menor ou igual $4 \mathrm{~mm}$ e maior ou igual a $2 \mathrm{~mm}$, tendo nessa circustância duas opções de tratamento, Toxina Botulínica Tipo A ou Reposicionamento Labial (Neto et al., 2017, Garber \& Salama, 1996; West, 2008).

Mesmo com esses critérios para a decisão do correto tratamento da condição de sorriso gengival, deve-se lembrar que a mesma pode apresentar uma ou mais etiologias distintas de forma simultânea com necessidade de uma ou mais intervenções para se alcançar o objetivo. Além disso, o trabalho interdisciplinar nessa condição se faz extremamente necessário e muito relevante para um resultado final mais satisfatório, lembrando que a saúde dos tecidos periodontais é fundamental para os passos de todo o tratamento de sucesso e a sua manutenção ao longo do tempo, pois saúde e estética são fatores que caminham lado a lado. Sendo assim, fica clara a importância de um plano de tratamento individualizado para cada paciente, dentro das condições e limitações de cado caso, levando sempre em consideração o sexo, a idade, a etnia e as expectativas estéticas e queixas de cada indivíduo (Neto et al., 2017, Seixas et al., 2011).

\section{Discussão}

A condição clínica de sorriso gengival é um aspecto que gera grande insatisfação e causa um impacto negativo na percepção de terceiros, que pode afetar a vida pessoal e profissional dos pacientes. Esses fatores 
indicam que a harmonia entre todas as estruturas da face são necessárias para alcançar uma estética favorável, sendo pertinente que a avaliação vá muito além da cavidade bucal. A literatura mostra que as etiologias do sorriso gengival são diversas podendo estar relacionadas no mesmo indivíduo, o que sugere, muitas vezes, um tratamento complexo (Rocha Neto \& Damin, 2009; Sarver \& Ackerman, 2003; Garber \& Salama, 1996; Batwa, 2018).

O sorriso gengival é considerado quando há exposição de gengiva de $2 \mathrm{~mm}$ ou mais, porém, quando comparamos as opiniões de cirurgiões dentistas e leigos essa característica não é avaliada da mesma forma, além disso, estudos mostram que uma certa quantidade de gengiva exposta ao sorrir é sinal de jovialidade sendo bem aceita esteticamente. Essa informação reforça o pensamento de que o ideal de estética é extremamente subjetivo e que nunca se deve esquecer de realizar o plano de tratamento junto ao paciente, além de respeitar as características individuais, como o sexo, a idade, a etnia e a cultura, não se prendendo a um padrão de beleza para todos. Essa questão é muito importante para que o resultado final seja agradável esteticamente ao paciente e esteja de acordo com os princípios de saúde e as limitações de cada técnica (Rivera et al., 2000; Owens et al., 2002; Tjan \& Miller, 1984; Arnett \& Bergman, 1993; Fowler, 1999; Zachrisson, 1998).

As etiologias dessa condição podem ser de origem Esquelética, Dental, Labial e Periodontal. Essa informação só confirma o fato de que é imprescindível um exame detalhado na avaliação facial, que sugere a análise dos seguintes pontos: distância interlabial em repouso, exposição dos incisivos superiores durante o repouso e a fala, arco do sorriso, proporção largura/comprimento dos incisivos superiores e características morfofuncionais do lábio superior, lembrando que assimetrias em distâncias lineares de até 3\% são aceitáveis. Sendo assim, fica evidente que a condição de sorriso gengival está ligada a tonicidade muscular em intensidade menor ou maior (Lu, 1995; Seixas et al., 2011; Duarte et al., 2009).

Da mesma forma que as etiologias, o tratamento do sorriso gengival é diversificado. A literatura sugere uma árvore de decisão para auxiliar na escolha que é composta por procedimentos como: Gengivectomia, Osteotomia, Cirurgia de Retalho Posicionado Apicalmente, Cirurgia Ortognática, Intrusão Ortodôntica, Toxina Botulínica e Reposicionamento Labial, realizados de forma isolada ou combinada. Vale ressaltar que os processos de Erupção Passiva e Ativa são fisiológicos e desejados, porém quando estes apresentam-se alterados se encaixam dentro das etiologias do sorriso gengival, onde é necessário realizar uma avaliação da gengiva queratinizada, do espaço biológico, da dimensão vertical e das proporções dentárias. Nesses casos, o tratamento tem o objetivo de reestabelecer a harmonia e otimização da estética, assim como a manutenção do espaço biológico (Neto et al., 2017; Garber \& Salama, 1996; Borghetti, 2002; Newman et al., 2016; Coslet et al., 1977; West, 2008).

Diante das informações apresentadas, não há dúvidas de que o sorriso gengival depende de uma série de características a serem analisadas que vão muito além dos tecidos periodontais e necessita de um trabalho interdisciplinar envolvendo diversas especialidades da odontologia. Apesar de existir no presente estudo um checklist estético e um plano previamente traçado para realizar um correto diagnóstico e posteriormente um tratamento, o mais indicado é seguir esses conceitos de forma personalizada para cada caso (Neto et al., 2017; Seixas et al., 2011).

\section{Considerações finais}

O desenvolvimento do presente estudo nos permite concluir qual o impacto do sorriso gengival na estética do sorriso, percebe-se como esse fato afeta negativamente a vida das pessoas em diversos aspectos, principalmente com a crescente demanda por padrões estéticos. Estudos mostram que essa condição causa impressões desfavoráveis por terceiros prejudicando as pessoas nos seus relacionamentos sociais assim como em sua vida profissional. Para reverter essas situações, se faz importante o conhecimento das etiologias do sorriso gengival, elas são variáveis e podem se apresentar de forma isolada ou combinadas entre si, sendo assim, o diagnóstico dessa condição exige uma análise criteriosa e um plano de tratamento individualizado para cada paciente, realizando uma avaliação facial completa, que relacione todas as estruturas constituintes, a fim de se alcançar a harmonização do rosto como um todo, o que, por sua vez, contribui de forma positiva para a estética do sorriso. Além disso, na maioria dos casos, para que seja possível alcançar um resultado satisfatório, o tratamento necessita de um trabalho interdisciplinar, podendo ser realizada mais de uma intervenção, que dependerá da necessidade de cada caso. 


\section{References}

Arnett, G. W., \& Bergman, R. T. 1993. Facial keys to orthodontic diagnosis and treatment planning. Part I. American Journal of Orthodontics and Dentofacial Orthopedics, 103(4), 299-312.

Batwa, W. 2018. The Influence of the Smile on the Perceived Facial Type Esthetics. BioMed Research International, $1-7$.

Borghetti, A. 2002. Cirurgia plástica periodontal. $1^{\text {a }}$ ed. Porto Alegre: Artmed.

Burstone, C. J. 1967. Lip posture and its significance in treatment planning. American Journal of Orthodontics, 53(4), 262-84.

Cohen, M. 2008. Interdisciplinary treatment planning: principles, design, implementation. $1^{\text {a }}$ ed. Seattle: Quintessence.

Coslet, J. G., Vanarsdall, R., \& Weisgold, A. 1977. Diagnosis and classification of delayed passive eruption of the dentogingival junction in the adult. The Alpha Omegan, 70(3), 24-28.

Cosendey, V. L. 2008. Avaliação do relacionamento entre o lábio superior e incisivos durante a fala e o sorriso. (Dissertação, Universidade do Estado do Rio de Janeiro). Rio de Janeiro: UERJ.

Chiche, G. J., \& Pinault, A. 1994. Esthetics of anterior fixed prosthodontics. Chicago, IL: Quintessence.

Desai, S., Upadhyay, M., \& Nanda, R. 2009. Dynamic smile analysis: changes with age. American Journal of Orthodontics and Dentofacial Orthopedics, 136(3), 310.e1-10.

Duarte, C. A., de Castro, M. V. M., \& Perreira, A. L. 2009. Cirurgia Periodontal Estética. In: Duarte, C. A. Cirurgia Periodontal - pré-protética e estética. $3^{\text {a }}$ ed. São Paulo: Santos, p. 343-406.

Fowler, P. 1999. Orthodontics and orthognatic surgery in the combined treatment of an excessive gummy smile. The New Zealand Dental Journal, 95(420), 53-4.

Garber, D. A., \& Salama, M. A. 1996. The aesthetic smile: diagnosis and treatment. Periodontology 2000, $11,18-28$.

Geron, S., \& Atalia, W. 2005. Influence of sex on the perception of oral and smile esthetics with different gingival display and incisal plane inclination. The Angle Orthodontist, 75(5), 778-784.

Kokich, V. G., Nappen, D. L, \& Shapiro, P. A. 1984. Gingival contour and clinical crown length: their effect on the esthetic appearance of maxillary anterior teeth. American Journal of Orthodontics, 86(2), 89-94.

Kumar, K. S. S., Deepika, M., Chandrasekaran, T. R., \& Janardhanam, P. 2007. Diagnosis and treatment planning of excessive gingival display-A review. Journal of Indian Orthodontic Society, 41(3), 112-115.

Kraut, R. E., \& Johnston, R. E. 1979. Social and emotional messages of smiling: an ethological approach. Journal of Personality and Social Psychology, 37(9), 1539-1553.

Levin, E. L. 1978. Dental esthetics and golden proportion. The Journal of Prosthetic Dentistry, 40(3), 244252.

Levine, R. A., \& Mcguire, M. 1997. The diagnosis and treatment of the gummy smile. Compendium of Continuing Education in Dentistry, 18(8), 757-762.

Legan H. L., \& Burstone, C. J. 1980. Soft tissue cephalometric analysis for orthognatic surgery. Journal of Oral Surgery, 38(10), 744-751.

Lu, K. H. 1965. Harmonic analysis of the human face. Biometrics, 21(2), 491-505.

Malkinson, S., Waldrop, T. C., Gunsolley, J. C., Lanning, S. K., \& Sabatini, R. 2013. The effect of esthetic crown lengthening on perceptions of a patient's attractiveness, friendliness, trustworthiness, intelligence and self-confidence. Journal of Periodontology, 84(8), 1126-1133.

Marckley, R. J. 1993. An evaluation of smiles before and after orthodontic treatment. The Angle Orthodontist, 63(3), 183-189.

Morais, A., Esteves, C. M., Dias, R. O., Rodrigues, J. A., Reis, A. F., \& Duarte, P. M. 2010. Cirurgia plástica periodontal para otimização de resultados estéticos na região anterior. Revista da Associação Paulista de Cirurgiões Dentistas, 64(2), 104-111.

Neto, J. B. C., Sirolli, M., Sekiguchi, R. T., Cabral, M., \& Silva, C. O. 2017. Correção do Sorriso Gengival. In: Pannuti, C. M., Villar, C. C., Romito, G. A., Saraiva, L., \& Holzheausen M. Estratégias Terapêuticas Atuais - No manejo da doença periodontal e peri-implantar. $1^{a}$ ed. São Paulo: Napoleão, p. 01-20. 
Newman, M. G., Takei, H. H., Klokkevold, P. R., \& Carranza, F. A. 2016. Carranza-Periodontia Clínica. 12a ed. Rio de Janeiro: Elsevier.

Owens, E. G., Goodacre, C. J., Loh, P. L., Hanke, G., Okamura, M., Jo, K. H., Muñoz, C. A., \& Naylor, W. P. 2002. A multicenter interracial study of facial appearance. Part 2: A comparison of intraoral parameters. The International Journal of Prosthodontics, 15(3), 283-288.

Pascotto, R. C., \& Moreira, M. 2005. Integração da Odontologia com a Medicina Estética: Correção do Sorriso Gengival. Revista Gaúcha de Odontologia, 53(3), 171-175.

Peck, S., Peck, L., \& Kataja, M. 1992. The gingival smile line. The Angle Orthodontist, 62(2), 91-100.

Pires, C. V., Souza, C. G. L. G., \& Menezes, S. A. F. 2010. Procedimentos Plásticos Periodontais em Paciente com Sorriso Gengival - Relato de Caso. Revista Periodontia, 20(1), 48-53.

Polo, M. 2008. Botulinum toxin type A (Botox) for the neuromuscular correction of excessive gingival display on smiling (gummy smile). American Journal of Orthodontics and Dentofacial Orthopedics, 133(2), 195-203.

Rivera, S. M., Hatch, J. P., Dolce, C., Bays, R. A., Van Sickels, J. E., \& Rugh, J. D. 2000. Patients' own reasons and patient-perceived recommendations for orthognatic surgery. American Journal of Orthodontics and Dentofacial Orthopedics, 118(2), 134-140.

Rocha Neto, O. M, \& Damin, L. 2009. Cirurgias Periodontais: Procedimentos relacionados à estética. Diálogos e Ciência - Revista da rede de ensino FTC, 3(9), 73-78.

Saba-Chujfi, E., Santos-Pereira, S. A. 2007. Periodontia: integração e resultados. São Paulo: Artes Médicas.

Sarver, D. M. 2004. Principles of cosmetic dentistry in orthodontics: Part 1. Shape and proportionality of anterior teeth. American Journal of Orthodontics and Dentofacial Orthopedics, 6(126), 749-753.

Sarver, D. M., \& Ackerman, M.B. 2003. Dynamic smile visualization and quantification: Part 2, smile analysis and treatment strategies. American Journal of Orthodontics and Dentofacial Orthopedics, 124(2), 116-127.

Seixas, M. R., Costa-Pinto, R. A., \& Araújo, T. M. 2011. Checklist dos aspectos estéticos a serem considerados no diagnóstico e tratamento do sorriso gengival. Dental Press Journal of Orthodontics, 16(2), 131-157.

Silva, C. O., Ribeiro-Junior, N. V., Campos, T. V. S., Rodrigues, J. G., \& Tatakis, D. N. 2013. Excessive gingival display: treatment by a modified lip repositioning technique. Journal of Clinical Periodontology, 40(3), 260-265.

Tjan, A. H., Miller, G. D., \& The, J. G. 1984. Some esthetic factors in a smile. The Journal of Prosthetic Dentistry, 51(1), 24-28.

Vig, R. G., \& Brundo, G. C. 1978. Kinetics of anterior tooth display. The Journal of Prosthetic Dentistry, 39(5), 502-504.

Zachrisson, B. U. 1998. Esthetic factors involved in anterior tooth display and smile: vertical dimension. Journal of Clininal Orthodontics, 32(7), 432-445.

Zangrando, M. S. R., Veronesi, G. F., Cardoso, M. V., Michel, R. C., Damante, C. A., Sant'ana, A. C. P, \& Greghi, S. L. A. 2017. Altered Active and Passive Eruption: A Modified Classification. Clinical Advances in Periodontics, 7(1), 51-56.

West JD. Framing your masterpiece: guidelines for treatment planning the ideal soft tissue framework. In: Cohen M. Interdisciplinary Treatment Planning: Principles, Design, Implementation. Chicago, IL: Quintessence Pub 2008: 333-374.

\section{Minicurrículo}

Nicole Fornari Sígolo. Graduada do Curso de Odontologia da Universidade São Francisco.

Emanuel Silva Rovai. Graduado em Odontologia pela Universidade Estadual Paulista (2012), Especialista em Periodontia pela Universidade de São Paulo (2016), Mestre em Odontologia Restauradora pela Universidade Estadual Paulista (2014) e Doutor em Periodontia pela Universidade de São Paulo (2018). 
Valdineia Maria Tognetti. Graduada em Odontologia pela Universidade São Francisco (2003), Especialista em Odontopediatria pela São Leopoldo Mandic (2009), Mestre em Saúde Coletiva em Odontologia na UNICAMP/FOP (2012), Doutoranda em odontologia com ênfase em Odontopediatria na UNICAMP/FOP.

Como citar: Sígolo, N.F., Rovai, E.S., \& Tognetti, V.M. 2021. Impacto da exposição gengival na estética do sorriso. Pubsaúde, 6, a138. DOI: https://dx.doi.org/10.31533/pubsaude6.a138

Recebido: 2 mar. 2021.

Revisado e aceito: 25 mar. 2021.

Conflito de interesse: os autores declaram, em relação aos produtos e companhias descritos nesse artigo, não ter interesses associativos, comerciais, de propriedade ou financeiros que representem conflito de interesse.

Licenciamento: Este artigo é publicado na modalidade Acesso Aberto sob a licença Creative Commons Atribuição 4.0 (CC-BY 4.0). 